\title{
Acute Phenylalanine/Tyrosine Depletion of Phasic Dopamine in the Rat Brain
}

\author{
Tatiana A. Shnitko ${ }^{1}$, Sarah C. Taylor ${ }^{1}$, Sierra J. Stringfield ${ }^{1,2}$, Shannon L. Zandy ${ }^{3}$, Roberto \\ U. Cofresi ${ }^{3}$, James M. Doherty ${ }^{3}$, William B. Lynch ${ }^{1}$, Charlotte A. Boettiger ${ }^{1,2,4}$, Rueben A. \\ Gonzales $^{3}$, and Donita L. Robinson ${ }^{1,2,5}$ \\ ${ }^{1}$ Bowles Center for Alcohol Studies, University of North Carolina, Chapel Hill, NC, USA \\ ${ }^{2}$ Neurobiology Curriculum, University of North Carolina, Chapel Hill, NC, USA \\ ${ }^{3}$ Division of Pharmacology and Toxicology, College of Pharmacy, The University of Texas at \\ Austin, Austin, TX, USA \\ ${ }^{4}$ Department of Psychology and Neuroscience, University of North Carolina, Chapel Hill, NC, \\ USA \\ ${ }^{5}$ Department of Psychiatry, University of North Carolina, Chapel Hill, NC, USA
}

\section{Abstract}

Rationale-Dopamine plays a critical role in striatal and cortical function, and depletion of the dopamine precursors phenylalanine and tyrosine is used in humans to temporarily reduce dopamine and probe the role of dopamine in behavior. This method has been shown to alter addiction-related behaviors and cognitive functioning presumably by reducing dopamine transmission, but it is unclear what specific aspects of dopamine transmission are altered.

Objectives-We performed this study to confirm that administration of an amino acid mixture omitting phenylalanine and tyrosine (Phe/Tyr[-]) reduces tyrosine tissue content in the prefrontal cortex (PFC) and nucleus accumbens (NAc), and to test the hypothesis that Phe/Tyr[-] administration reduces phasic dopamine release in the NAc.

Methods-Rats were injected with a Phe/Tyr[-] amino acid mixture, a control amino acid mixture, or saline. High-performance liquid chromatography was used to determine the concentration of tyrosine, dopamine, or norepinephrine in tissue punches from the PFC and ventral striatum. In a separate group of rats, phasic dopamine release was measured with fast-scan cyclic voltammetry in the NAc core after injection with either the Phe/Tyr[-] mixture or the control amino acid solution.

Corresponding author: Donita L. Robinson, PhD, Bowles Center for Alcohol Studies, CB \#7178, University of North Carolina, Chapel Hill, NC 27599-7178; DLR@unc.edu; Phone: 919-966-9178 ; Fax: 919-966-5679.

Author Contribution

DLR and CAB conceptualized the experiments. TAS, SCT, DLR and WL collected and analyzed the voltammetric data. SJS, SZ, RUC, JMD and RAG set up HPLC procedures and collected and analyzed the tissue content data. DLR, TAS, SJS, SZ, CAB and RAG interpreted the data and prepared the manuscript.

Notes: The authors declare no competing financial interest. 
Results-Phe/Tyr[-] reduced tyrosine content in the PFC and NAc, but dopamine and norepinephrine tissue content were not reduced. Moreover, Phe/Tyr[-] decreased the frequency of dopamine transients, but not their amplitude, in freely-moving rats.

Conclusions-These results indicate that depletion of tyrosine via Phe/Tyr[-] decreases phasic dopamine transmission, providing insight into the mechanism by which this method modifies dopamine-dependent behaviors in human imaging studies.

\section{Keywords}

Dopamine; norepinephrine; tyrosine; fast-scan cyclic voltammetry; nucleus accumbens; prefrontal cortex; depletion

\section{Introduction}

The critical role of dopamine in striatal and cortical function has been emphasized in numerous studies. In human subjects, the tools available to probe dopamine function are limited. One method currently used to investigate dopamine's role in human behaviors is acute phenylalanine/tyrosine depletion (Moja et al., 1996, Leyton et al., 2000b, Leyton et al., 2004). With this method, subjects drink amino-acid (AA) mixtures either containing or lacking the catecholamine precursors tyrosine (Tyr) and phenylalanine (Phe). Ingestion of AA mixtures alters the level of individual AA species in systemic circulation and promotes protein synthesis by replenishing AA pools. Importantly, tryptophan and the branched-chain AAs (valine, leucine, and isoleucine) compete with Phe and Tyr for active transport across the blood-brain barrier. The Phe/Tyr deficient (Phe/Tyr[-]) mixture, as compared to the balanced control (BAL) mixture, results in lower Phe/Tyr levels relative to other AAs in circulation and, due to competition, reduced Phe/Tyr transport across the blood-brain barrier. Subsequently, the reduced availability of both catecholamine precursors would be expected to decrease catecholamine synthesis in the brain. That said, evidence to date indicates that Phe/Tyr[-] administration alters dopamine but not norepinephrine release (Sheehan et al., 1996, McTavish et al., 1999a, McTavish et al., 1999b, Harmer et al., 2001, McTavish et al., 2001).

In humans, Phe/Tyr[-] treatment reduces alcohol self-administration (Leyton et al., 2000a, Barrett et al., 2008), dampens mood and motivational state (Leyton et al., 2000b, Cawley et al., 2013), decreases cigarette smoking (Venugopalan et al., 2011), and can impair executive function and reinforcement learning (Kelm and Boettiger, 2013, 2015). Moreover, positron emission tomography (PET) has been used to determine that dopamine D2 receptor availability was greater following Phe/Tyr[-] both at baseline (Montgomery et al., 2003) and after an amphetamine challenge (Leyton et al., 2004), indicating that Phe/Tyr[-] administration reduced basal and stimulated extracellular dopamine as compared to ingestion of the BAL solution. However, PET imaging has limited spatial and temporal resolution, and to date PET imaging of the dopamine system in humans has been limited to radioligands that allow assessment only of striatal dopamine signaling. As such, neural localization of the various behavioral effects of Phe/Tyr[-] treatment in humans remains unclear. 
More precise tools, such as microdialysis and fast-scan cyclic voltammetry (FSCV), are available in animal models to directly measure extracellular dopamine concentrations within the context of both pharmacological manipulations and behavioral paradigms. Therefore, combining acute Phe/Tyr[-] with invasive neurochemical methods in animal models provides a unique opportunity to identify and more precisely localize the physiological and neurochemical consequences of Phe/Tyr[-] treatment, giving new insight to how this manipulation impacts brain function. To this end, several studies have documented consequences of Phe/Tyr[-] administration in rats. Intragastric administration of a Phe/Tyr[-] AA mixture decreases Tyr levels and the rate of Tyr hydroxylation in the central nervous system (Fernstrom and Fernstrom, 1995). Moreover, intraperitoneal Phe/Tyr[-] administration reduces Tyr tissue content and several indices of catecholamine function in both frontal cortex and striatum, including amphetamine-induced release of dopamine and desipramine-induced enhancement of dopamine and norepinephrine concentration (e.g., McTavish et al., 1999b, Bongiovanni et al., 2008). To date, the effects of Phe/Tyr[-] treatment on extracellular dopamine concentrations in the brain have been measured with microdialysis, a technique suited to monitor tonic changes in dopamine over several minutes to hours. However, dopamine release also occurs on a subsecond timescale; these fluctuations in dopamine release are termed dopamine transients and they reflect the brief efflux of neurotransmitter following dopamine neuron burst firing (Sombers et al., 2009). Both tonic and phasic dopamine release are involved in behavioral control, but these two modes differentially activate D1 and D2 receptors. Low concentration, tonic dopamine is thought to provide "tone" to high-affinity D2 receptors, while high concentration dopamine transients are sufficient to activate low-affinity D1 receptors (Dreyer et al., 2010). Critically, no published studies to date have investigated the effect of Phe/Tyr[-] treatment on phasic dopamine transmission, which is ideally accomplished via FSCV (Robinson et al., 2008) due to its temporal and spatial resolution.

To address this gap in the literature, the present study used FSCV to determine the effects of Phe/Tyr[-] administration on phasic dopamine release in freely-moving rats. To our knowledge, spontaneous dopamine transients have not been reported with FSCV in the prefrontal cortex (PFC) and would not be electrochemically distinguished from norepinephrine (e.g., Shnitko and Robinson, 2014); thus, we targeted the nucleus accumbens (NAc), where dopamine transients have been well described. We hypothesized that acute Phe/Tyr depletion would diminish newly synthesized dopamine and thereby reduce the frequency and size of spontaneous dopamine transients in NAc of rats. To address our hypothesis, we administered an AA mixture that either contained (BAL) or omitted (Phe/ Tyr[-]) Phe and Tyr. We also confirmed that Tyr was depleted in the NAc to complement the FSCV experiment, as well as in the PFC, which is a region of interest in many human studies using acute Phe/Tyr depletion. Finally, we determined the tissue content of dopamine and norepinephrine in the NAc and the PFC in order to assess whether acute Phe/Tyr depletion significantly altered storage pools of catecholamine in these regions. 


\section{Methods}

Animals

Adult, male Sprague Dawley rats $(\mathrm{n}=18,277 \pm 17 \mathrm{~g}$ in Experiment 1 , and $\mathrm{n}=12,330 \pm 11 \mathrm{~g}$ in Experiment 2) were purchased from Harlan Laboratories (Frederick, MD) and pair-housed in a temperature- and humidity-controlled room on a 12-h light-dark schedule with food and water ad libitum. All procedures involving laboratory animals complied with the Guide for Care and Use of Laboratory Animals and were approved by the Institutional Animal Care and Use Committee of the University of North Carolina at Chapel Hill.

\section{Amino Acid Solutions}

The AA solutions were adapted from McTavish et al. (1999b) and prepared as follows. For the Phe/Tyr[-] mixture, the following AAs were added to $5 \mathrm{~mL} 1 \mathrm{~N} \mathrm{NaOH}: 100 \mathrm{mg}$ methionine, $200 \mathrm{mg}$ threonine, $50 \mathrm{mg}$ tryptophan, $350 \mathrm{mg}$ lysine, $415.7 \mathrm{mg}$ valine methyl ester HCL. This solution was stirred for $5 \mathrm{~min}$, and then the following AAs were added: $415.5 \mathrm{mg}$ isoleucine methyl ester $\mathrm{HCl}$ and $623.2 \mathrm{mg}$ leucine methyl ester $\mathrm{HCl}$. The solution was brought to a final volume of $12.3 \mathrm{~mL}$ using distilled water, and the $\mathrm{pH}$ was adjusted to 7.4 with $1 \mathrm{~N} \mathrm{NaOH}$. For the balanced AA mixture (BAL), the depletion mixture was prepared as stated above and brought to a final volume of $6.5 \mathrm{~mL}$. In a separate vial, $250 \mathrm{mg}$ of tyrosine methyl ester $\mathrm{HCl}$ and $250 \mathrm{mg}$ phenylalanine were added to $9 \mathrm{~mL}$ of distilled water and stirred for $5 \mathrm{~min}$. The two solutions were combined and stirred for an additional $10 \mathrm{~min}$, and then the $\mathrm{pH}$ was adjusted to 7.4 with $1 \mathrm{~N} \mathrm{NaOH}$. Solutions were made fresh daily and in two batches (one for each injection), immediately before the injection took place. After solvation, solutions were continuously mixed on a stir plate until time of injection. Isoleucine methyl ester HCL was purchased from Tokyo Chemical Industry Co. (Tokyo, Japan) and all other AAs were obtained from Sigma-Aldrich (St. Louis, Missouri). All rats were injected i.p. with a total volume $6.74 \mathrm{~mL} / \mathrm{kg}$ to deliver $1 \mathrm{~g} / \mathrm{kg}$ of AAs per injection.

\section{Experiment 1. Effect of Phe/Tyr[-] on tissue tyrosine and catecholamine content in forebrain tissue}

Tissue dissection, homogenate deproteinization and derivatization-Rats were injected with either the Phe/Tyr[-], BAL, or an equivalent volume of saline both 2 hours before and 1 hour before unanesthetized decapitation (see Figure 1A for timeline). The brains were quickly extracted and frozen in isopentane cooled with dry ice. Frozen brains were sliced on a cryostat and tissue was collected by using $1 \mathrm{~mm}$ tissue punches (Miltex, York, PA). Punches targeted the PFC, specifically the medial prefrontal and orbitofrontal cortices, and the ventral striatum, specifically the NAc core and shell. Tissue samples were weighed and $500 \mu \mathrm{l}$ of $0.1 \mathrm{~N}$ perchloric acid was added to each sample before homogenization using a sonicator probe. Samples were centrifuged at $13000 \mathrm{rpm}$ for $15 \mathrm{~min}$ at $4^{\circ} \mathrm{C}$. The supernatant was transferred to a separate tube and stored at $-80^{\circ} \mathrm{C}$ or on dry ice until further processing.

After thawing, $100 \mu \mathrm{L}$ of tissue homogenate or standard was added to $900 \mu \mathrm{L}$ of $1 \mathrm{M} \mathrm{HClO}_{4}$. Either $1000 \mu \mathrm{L}$ or $1100 \mu \mathrm{L}$ of $1 \mathrm{M} \mathrm{KOH}$ was added to adjust the $\mathrm{pH}$ for catecholamine or Tyr 
analysis, respectively. Following precipitation of $\mathrm{KClO}_{4}$, the solution was filtered through a $0.2 \mu \mathrm{m}$ nylon membrane. For catecholamine analysis, either 5 or $10 \mu \mathrm{L}$ was manually injected into a high performance liquid chromatography (HPLC) system. For Tyr analysis, $50 \mu \mathrm{L}$ of prepared sample or standard was added to $50 \mu \mathrm{L}$ of $0.1 \mathrm{M}$ sodium tetraborate buffer (pH 10.4). Electroactive derivatives were formed by adding $2 \mu \mathrm{L}$ of reagent and then incubating at room temperature for 10 minutes, and $15 \mu \mathrm{L}$ of the reaction mixture were manually injected into a separate HPLC system. The derivatizing reagent was prepared daily using $11 \mathrm{mg}$ of $o$-pthaldialdehyde, $250 \mu \mathrm{L}$ of ethanol, $250 \mu \mathrm{L}$ of $1 \mathrm{M}$ sodium sulfite, and 4.5 $\mathrm{mL}$ of $0.1 \mathrm{M}$ sodium tetraborate buffer ( $\mathrm{pH}$ 10.4), based on the methods of Smith and Sharp (1994).

Chromatography-For catecholamine analysis, reversed-phase HPLC system consisted of a Luna ${ }^{\circledR} 3 \mu \mathrm{m} \mathrm{C18(2)} 100 \AA$, 150 x 2 mm column (Phenomenex, Torrance, CA) and a SenCell2 flow cell with salt bridge reference electrode $(\mathrm{Ag} / \mathrm{AgCl})$ using an oxidizing potential of $+450 \mathrm{mV}$ (Antec Leyden, Netherlands). Degassed mobile phase $(0.50 \mathrm{~g}$ octanesulfonic acid, $0.05 \mathrm{~g}$ decanesulfonic acid, $0.13 \mathrm{~g}$ ethylenediaminetetraacetic acid, $11.08 \mathrm{~g} \mathrm{NaH}_{2} \mathrm{PO}_{4}, 100-150 \mathrm{~mL}$ methanol in $1 \mathrm{~L}$ deionized water; $\mathrm{pH}$ adjusted to 5.6 before addition of methanol) was delivered to the column at a flow rate of $0.28 \mathrm{ml} / \mathrm{min}$ using a LC110S pump (Antec Leyden). The column and electrochemical cell were housed in an INTRO controller (Antec Leyden) with oven temperature set to $45^{\circ} \mathrm{C}$ equipped with an 8125 manual injector (Rheodyne, Cotati, CA). EZChrom Elite chromatography software (Agilent, Wilmington, DE) was used for analysis of the chromatograms.

For Tyr analysis, reversed-phase HPLC system consisted of a Luna ${ }^{\circledR} 3 \mu \mathrm{m} \mathrm{C18(2)} 100 \AA$, $150 \times 2 \mathrm{~mm}$ column (Phenomenex) and VT-03 electrochemical flow cell with salt bridge reference electrode $(\mathrm{Ag} / \mathrm{AgCl})$ using an oxidizing potential of $+700 \mathrm{mV}$ (Antec Leyden). Degassed mobile phase $\left(0.186 \mathrm{~g}\right.$ ethylenediaminetetraacetic acid, $15.60 \mathrm{~g} \mathrm{NaH}_{2} \mathrm{PO}_{4}, 170$ $200 \mathrm{~mL}$ methanol in $1 \mathrm{~L}$ deionized water; $\mathrm{pH}$ adjusted to 4.5 before addition of methanol) was delivered to the column at a flow rate of $0.32 \mathrm{ml} / \mathrm{min}$ using a LC110 pump (Antec Leyden). A DECADE II controller (Antec Leyden) with oven temperature set to $45^{\circ} \mathrm{C}$ and 7125 manual injector (Rheodyne) were used. Chromeleon chromatography software (Thermo Scientific, Sunnyvale, CA) was used for analysis of the chromatograms.

External standards were used to quantify analyte concentrations. Catecholamine stocks were prepared in $0.1 \mathrm{M} \mathrm{H}_{3} \mathrm{PO}_{4}$. Tyr stock was prepared in purified water (Milli-Q®, EMD Millipore, Billerica, MA). Reported values are derived from chromatograms in which the analyte peaks had signal-to-noise ratios above 3 .

Statistical analysis-One NAc sample was excluded from the Tyr analysis due to poor chromatography. Two samples from PFC were identified as outliers (z-score \pm 3 ) and excluded from dopamine and norepinephrine analyses. Separate one-way ANOVA were conducted on dopamine, norepinephrine or Tyr content ( $\mathrm{pg} / \mathrm{mg}$ wet tissue) in NAc or PFC looking at the effect of treatment (3 levels: saline, BAL, Phe/Tyr[-]) using SPSS Statistics (version 21, IBM Corp., Armonk, NY). T-tests with Bonferroni corrections were used for post-hoc analyses. Only results with $p<0.05$ were considered significant. Data are presented as mean $\pm \mathrm{SEM}$, and group numbers are stated in figure captions. 


\section{Experiment 2. Effect of Phe/Tyr[-] on spontaneous dopamine transients in the NAc}

Surgery-Rats were surgically prepared for FSCV as previously described (Robinson et al., 2009, Shnitko and Robinson, 2015). In brief, rats were anesthetized with $2 \%$ isofluorane and placed in a stereotaxic frame on a heated pad. The skull was exposed and holes were drilled in the skull for placement of a stimulation electrode aimed at the ventral tegmental area (from bregma, AP: $-5.2 \mathrm{~mm}$, ML: $+1.0 \mathrm{~mm}, \mathrm{DV}-8.5 \mathrm{~mm}$ ) and a guide cannula (BAS Bioanalytical Systems, Inc, West Lafayette, IN) aimed above the NAc (AP: $1.8 \mathrm{~mm}$, ML: $+1.5 \mathrm{~mm})$. An $\mathrm{Ag} / \mathrm{AgCl}$ reference electrode was placed in the left hemisphere, contralateral to the guide cannula. All elements were secured with stainless steel screws and dental acrylic. After surgery, rats were given ibuprofen and allowed 4-5 days to recover.

Fast-scan cyclic voltammetry-Spontaneous dopamine transients in the NAc of awake rats were measured using FSCV at carbon-fiber microelectrodes as previously described (Robinson et al., 2009, Shnitko and Robinson, 2015). Animals were habituated to the experimental chamber and the tether one day prior to recording. On the day of the experiment, a carbon-fiber electrode was lowered into the NAc via the guide cannula and a triangle waveform $(-0.4 \mathrm{~V}$ to $1.3 \mathrm{~V}$ to $-0.4 \mathrm{~V}$ at $400 \mathrm{~V} / \mathrm{s}$ vs the $\mathrm{Ag} / \mathrm{AgCl}$ reference electrode) was applied at $60 \mathrm{~Hz}$ for at least 20 minutes to condition the microelectrode (Hermans et al., 2008). Next, the same waveform application frequency was reduced to 10 $\mathrm{Hz}$ to allow dopamine measurement every $100 \mathrm{~ms}$. Electrical stimulation $(60 \mathrm{~Hz}, 24$ pulses and $124 \mu \mathrm{A}$ ) was delivered to the ventral tegmental area in order to evoke dopamine in the $\mathrm{NAc}$ as a positive control to ensure that the carbon-fiber microelectrode was in a site that supported dopamine release (Robinson et al., 2003). Once reliable evoked (signal-to-noise > 20) and/or spontaneous (signal-to-noise > 5) dopamine release was obtained, the carbon fiber was secured in place and voltammetric recording began.

Experimental procedure-The experimental procedure is illustrated in Figure 1B. Initially, basal level measurements were collected for $20 \mathrm{~min}$ to observe spontaneous dopamine release events (i.e., dopamine transients). After baseline collection, rats received the first injection of Phe/Tyr[-] or BAL, followed by the second injection one hour later. Data collection continued for two hours after the second injection and the target window for analysis, based on previous studies (McTavish et al., 1999b), began one hour after the second injection. Thus, the experiment was conducted over 3 hours and 20 min. After the experiments, carbon-fiber electrodes were calibrated using known concentrations of dopamine $(0.5 \mu \mathrm{M}$ and $1.0 \mu \mathrm{M})$ in TRIS buffer $\left(2.5 \mathrm{mM} \mathrm{KCl}, 2.4 \mathrm{mM} \mathrm{CaCl}_{2}, 1.2 \mathrm{mM}\right.$ $\mathrm{MgCl}_{2}, 2.0 \mathrm{mM} \mathrm{Na}_{2} \mathrm{SO}_{4}, 1.2 \mathrm{mM} \mathrm{NaH} \mathrm{PO}_{4}, 15 \mathrm{mM}$ TRIS HCl, $126 \mathrm{mM} \mathrm{NaCl}, \mathrm{pH}=7.4$ ).

Histology-Animals were perfused through the heart with $0.9 \%$ saline and then $10 \%$ formaldehyde. The brains were removed and stored in a freezer at $-80^{\circ} \mathrm{C}$ until sliced with a cryostat in 50- $\mu \mathrm{m}$ coronal sections. The slices were stained with thionin and examined under a microscope to determine the placements of the guide cannulae.

Data analysis-FSCV data were analyzed by using a template match to a known cyclic voltammogram of dopamine in TarHeel CV software (Department of Chemistry, University of North Carolina at Chapel Hill) as previously described (Robinson et al., 2003). 
Voltammetric signals collected every $100 \mathrm{~ms}$ were presented as cyclic voltammograms where currents resulting from oxidation and reduction of electroactive compounds are plotted versus applied potential. Each cyclic voltammogram was compared with a known voltammogram of dopamine (or template) and the correlation between the experimental voltammogram and the template was assessed. Voltammetric signals with a correlation $\geq 0.866\left(\mathrm{r}^{2}>0.7\right)$ were considered dopaminergic. Next, the maximal amplitude of each transient was converted to $[\mathrm{DA}]_{\max }$ by using calibration factors. Only transients that were $\geq 5$ times the root mean square of the noise, as determined by the background scans for that transient, were counted as dopamine transients. The number of transients per minute and $[D A]_{\max }$ of the signals were used as parameters of dopaminergic activity at baseline and after injection of AA mixtures.

The present study measured frequency and amplitude of dopamine transients, similar to previous studies (Robinson and Wightman, 2004, Robinson et al., 2011, Howard et al., 2013). The effect of Phe/Tyr[-] on dopamine transient frequency was analyzed with two-way repeated-measures (RM) ANOVA of treatment (between-subject: BAL, Phe/Tyr[-]) by time (within-subject: baseline, three 20-min bins during the target window) using SigmaPlot (Systat Software, San Jose, CA). As the data were not normally distributed per the ShapiroWilk Normality Test, they were transformed by rank before analysis so they fit the assumptions of the parametric test. Post-hoc comparisons were calculated by the HolmSidak method. The effect of Phe/Tyr[-] on the distribution of amplitudes (or [DA $]_{\max }$ ) of dopamine transients was analyzed with a Rao-Scott Chi-Square test with treatment as a between-subject variable (BAL, Phe/Tyr[-])with multiple observations made within each rat. $[D A]_{\max }$ values were divided into 5 amplitude ranges or bins. Only results with $p<0.05$ were considered significant. Data are presented as mean \pm SEM, and group numbers are stated in figure captions.

\section{Results}

The injection regimen was based on McTavish et al. (1999b), in which the AA load was delivered in two i.p. injections, one hour apart. The critical comparison was between groups receiving Phe/Tyr[-] or the BAL control; however, for Experiment 1, we included an additional control group that received equivalent volumes of saline to determine any effects of AA load per se. The experimental procedure is illustrated in Figure 1. Tissue harvesting (Experiment 1) occurred one hour after the second injection. FSCV measurements (Experiment 2) spanned the pre-injection period through two hours after the second injection; however, only data from the pre-injection period (baseline) and 1-2 hours after the second injection were used for the analysis, as a previous microdialysis study found a significant effect of the Phe/Tyr[-] in the NAc of rats during the second hour after the second injection (McTavish et al. 1999).

\section{Experiment 1. Effect of Phe/Tyr[-] on tissue tyrosine and catecholamine content in forebrain tissue}

To confirm that Phe/Tyr[-] used in the current study resulted in Tyr depletion in tissue, we examined Tyr content with HPLC and electrochemical detection in tissue punches from NAc 
and PFC, as shown in Figure 2A. We compared tissue in rats receiving Phe/Tyr[-] to those receiving BAL or saline injections. Administration of Phe/Tyr[-] reduced tissue levels of Tyr to $<20 \%$ of levels in BAL rats and $<30 \%$ of levels in Saline rats, as shown in Figure 3A. Specifically, one-way ANOVA yielded differences in Tyr tissue content among treatment groups in the NAc $\left(\mathrm{F}_{2,16}=53.1 ; \mathrm{p}<0.001\right)$ and $\mathrm{PFC}\left(\mathrm{F}_{2,17}=35.8 ; \mathrm{p}<0.001\right)$. Post-hoc comparisons revealed that Tyr content in the depletion group was significantly reduced compared to both BAL and Saline groups in both forebrain regions $(\mathrm{p}<0.01)$. Additionally, Tyr content within the PFC was significantly higher in the BAL group compared to the Saline group $(\mathrm{p}<0.05)$.

To determine whether the reduction in Tyr led to reduced tissue content of dopamine or norepinephrine, separate aliquots of the same tissue samples were analyzed for catecholamine content. There were no significant differences in dopamine tissue content between groups in either NAc $\left(F_{2,17}=0.25 ; n s\right)$ or PFC $\left(F_{2,16}=0.71 ; n s\right)$ (Figure 3B). Similarly, no differences were observed in norepinephrine tissue content in PFC $\left(\mathrm{F}_{2,16}=1.29 ; \mathrm{ns}\right)$. There was a trend toward group differences in NAc norepinephrine content (Figure $3 \mathrm{C}$ ), but this did not reach statistical significance $\left(\mathrm{F}_{2,17}=3.21 ; \mathrm{p}=0.07\right)$. Therefore, although Tyr was depleted in rat forebrain after administration of Phe/Tyr[-], this depletion did not result in significantly lower catecholamine levels in tissue.

\section{Experiment 2. Effect of Phe/Tyr[-] on spontaneous dopamine transients in the NAc}

We used FSCV to evaluate effects of Phe/Tyr[-] and BAL on naturally occurring dopamine transients in the NAc. Recording sites are depicted in Figure 2B. We found that Phe/Tyr[-] administration reduced the frequency of dopamine transients. An example from an individual rat is shown in Figure 4, where the voltammetric signal obtained in the first 8 seconds of the baseline period is compared to the signal in the first 8 seconds of the target window (60-120 min after the second injection of Phe/Tyr[-]) in the same rat. The color plots display oxidative currents (when applied potential ramps from -0.4 to $+1.3 \mathrm{~V}$ ) and reductive currents (when applied potential decreases from +1.3 to $-0.4 \mathrm{~V}$ ) expressed in color, with applied potential on the $y$-axis and time on the $\mathrm{x}$-axis. Above the color plot, current at the oxidative potential of dopamine $(\sim 0.65 \mathrm{~V}$ vs $\mathrm{Ag} / \mathrm{AgCl}$ reference electrode) is extracted and converted to concentration, with dopamine transients indicated by the asterisk and confirmed via the cyclic voltammogram. While dopamine transients were evident at baseline, they were largely absent after Phe/Tyr[-].

We next compared the number of transients during the baseline period to the three 20-min bins of the target window (60-120 min after the second injection), as shown in Figure 5A. Basal rates of dopamine transients were $1.9 \pm 0.4$ per minute in the BAL group and $2.5 \pm 0.7$ in the Phe/Tyr[-] group. Phe/Tyr[-] decreased the number dopamine transients by $50-75 \%$ from baseline levels, as well as compared to the BAL group. A two-way RM ANOVA revealed main effects of group $\left(\mathrm{F}_{3,30}=5.0, \mathrm{p}<0.05\right)$ and time $\left(\mathrm{F}_{3,30}=7.2, \mathrm{p}<0.001\right)$ and a significant group by time interaction $\left(\mathrm{F}_{3,30}=4.3, \mathrm{p}<0.05\right)$. Post-hoc comparisons found fewer dopamine transients in the Phe/Tyr[-] group during each 20-min bin of the target window compared to baseline in the same rats (all $\mathrm{t}>4.2$, all $\mathrm{p}<0.001$ ) as well as compared to BAL rats during the target window (all $\mathrm{t}>2.3$, all $\mathrm{p}<0.05$ ). 
We hypothesized that Phe/Tyr[-] would also reduce the amplitude of phasic dopamine events. To assess this, we evaluated the distribution of transient amplitudes within the 60min target window by plotting the proportion of transients ranging from $<10 \mathrm{nM}$ to $>40 \mathrm{nM}$ [DA $]_{\max }$ (e.g., Robinson and Wightman, 2004, Robinson et al., 2011). Overall, Phe/Tyr[-] did not alter the distribution of [DA $]_{\max }$ as compared to BAL (Figure 5B). A Rao-Scott ChiSquare test did not yield a significant difference in amplitude between the BAL and Phe/ Tyr[-] groups (Rao-Scott $\lambda^{2}{ }_{4,44}=2.53$, p $>0.05$ ). Thus, while Phe/Tyr[-] effectively diminished the frequency of phasic dopamine release events, the amplitude of those transients was not diminished.

\section{Discussion}

Dietary Tyr depletion is a method to temporarily dampen dopamine function in humans and is increasingly used in clinical and basic science. While PET studies have suggested that extracellular dopamine concentrations are generally reduced (Montgomery et al., 2003, Leyton et al., 2004), the consequences of Tyr depletion on phasic dopamine release events are unknown. While the recording of dopamine transients using FSCV in the human brain is currently under development (Kishida et al., 2011), the use of FSCV in rodents is decades ahead and available for immediate use. The present study used a rat model to determine the impact of acute tyrosine depletion via Phe/Tyr[-] on spontaneous dopamine transients. We confirmed that Phe/Tyr[-] effectively depleted Tyr in dopamine terminal regions. We also measured phasic dopamine fluctuations in the NAc core in real time with FSCV. We found that Phe/Tyr[-] reduced the frequency of dopamine transients by $50-75 \%$, while rates of transients in BAL rats were unchanged. This reduction is similar to reductions in spontaneous dopamine transients observed after dopamine lesion due to methamphetamine toxicity (Howard et al., 2013). However, whereas the dopamine lesion also reduced the amplitude of dopamine transients (Howard et al., 2013), the temporary Phe/Tyr[-] used here did not reduce $[\mathrm{DA}]_{\max }$ of the remaining dopamine transients relative to BAL controls. Overall, these data show that Tyr depletion via a Phe/Tyr deficient AA load reduces spontaneous, phasic dopamine release and, by extension, may impact behaviors that require phasic signaling, such as reward learning.

The present study employed FSCV-which has the chemical, spatial and temporal resolution to distinguish individual dopamine release events - to monitor dopamine transients in the NAc core, as phasic dopamine in this brain region is well described. An accumulating amount of literature has characterized phasic dopamine transients as neural correlates of reward-associated learning, including salient sensory processing, such as predictive cues and prediction error (for review, see Robinson et al., 2008, Willuhn et al., 2010, Schultz, 2016). Thus, the finding that Phe/Tyr[-] reduces phasic dopamine release may be important in the interpretation of human-subject studies using Phe/Tyr depletion. For example, the finding that Phe/Tyr[-] impairs learning from both reward and punishment in late adolescents/ emerging adults, but not adults (Kelm and Boettiger, 2015), suggests changes in dopamine transients across the adolescent-adult transition, a possibility supported by data from adolescent and adult rats (Robinson et al., 2011). The direct association between dopamine transients that are time-locked to specific discrete cues or rewards in trained rats (e.g., Day et al., 2007) and the "background" of dopamine transients over a broader timeframe as 
measured here is not clear, as most studies do not include both measurements. However, we have previously reported that the frequency and amplitude of dopamine transients increases upon presentation of salient stimuli (Robinson et al., 2002, Robinson and Wightman, 2004, Robinson et al., 2011), which is consistent with the idea that when reward learning is likely to occur, phasic dopamine transmission is enhanced. Conversely, it is also plausible that when phasic dopamine is decreased, as herein or with more permanent disruptions of dopamine (Howard et al., 2011, Howard et al., 2013), it may explain effects on cognition reported in the corresponding human literature (e.g., Volkow et al., 2001, Kelm and Boettiger, 2013, 2015).

Previous studies have used microdialysis to assess Phe/Tyr[-] effects on tonic dopamine transmission in rat models. Interestingly, initial studies reported that Phe/Tyr[-] administration failed to reduce basal concentrations of extracellular dopamine in prefrontal cortex and dorsal striatum (McTavish et al., 1999b, Jaskiw and Bongiovanni, 2004, Jaskiw et al., 2005, Jaskiw et al., 2006, Bongiovanni et al., 2008). However, later studies reported a decrease in tonic dopamine in the dorsal striatum 30-60 min (Brodnik et al., 2013) or 100120 min (Le Masurier et al., 2006) after the second Phe/Tyr[-] injection, with no concomitant changes in basal dopamine in the prefrontal cortex (Brodnik et al., 2013). A lack of Phe/Tyr[-] effect on basal dopamine levels is not unexpected when one considers that dopamine terminals maintain extracellular dopamine levels through a balance between release and uptake mechanisms, and are known to robustly maintain dopamine tone even after sizable dopamine lesions (Garris et al., 1997). However, when dopamine concentrations were augmented by amphetamine (McTavish et al., 1999b, Le Masurier et al., 2006), catecholamine uptake blockers (Bongiovanni et al., 2008), antipsychotics (Jaskiw and Bongiovanni, 2004, Jaskiw et al., 2005, Jaskiw et al., 2006) or electrical stimulation of the medial forebrain bundle (Le Masurier et al., 2013), Phe/Tyr[-] administration reduced subsequent dopamine concentrations compared to controls. While these microdialysis studies show that blocking uptake or enhancing release reveals the vulnerability of tonic dopamine levels to Phe/Tyr[-], microdialysis does not have the sensitivity to determine whether phasic dopamine events are altered by Phe/Tyr[-] (Borland et al., 2005), as microdialysis samples are collected over several minutes and millimeters of tissue.

We confirmed that the Phe/Tyr[-] injection regimen depleted Tyr in the brain by analyzing tissue content in tissue punches from the PFC and NAc. In both brain regions, at $60 \mathrm{~min}$ after the second injection, Tyr was depleted to less than $20 \%$ of BAL levels in the Phe/Tyr[-] group. In contrast, dopamine and norepinephrine tissue content was not significantly altered at this time point, consistent with prior data (McTavish et al., 1999b). This finding is not surprising, considering that Tyr depletion would preferentially deplete vesicles with newly synthesized dopamine and norepinephrine while approximately $80 \%$ of catecholamines are thought to be stored in reserve pools (Alabi and Tsien, 2012). We observed that Phe/Tyr[-] slightly lowered norepinephrine content in the NAc (although this decline did not reach statistical significance), in contrast to prior research suggesting that norepinephrine release is less sensitive to disruptions in synthesis via inhibition of Tyr hydroxylase or Phe/Tyr[-] than dopamine release (Sanghera et al., 1979, McTavish et al., 1999a, Park et al., 2011). These findings have been interpreted as evidence that the readily releasable pool of norepinephrine is less dependent on newly synthesized norepinephrine and less distinct from other storage 
pools (McMillen et al., 1980), and it should be noted that no reduction in norepinephrine was observed in PFC tissue after Phe/Tyr[-] in the present study. However, dopamine is several-fold more abundant in the NAc than norepinephrine, and one speculation is that noradrenergic terminals rely on dopamine uptake through the norepinephrine transporter to replenish norepinephrine in vesicles; thus, the reduction in released dopamine may have been reflected in reduced norepinephrine tissue content. However, this observation should be repeated before it is concluded that there are regional differences in the effects of Phe/Tyr[-] on catecholamine tissue content.

Some caveats to this study should be acknowledged. First, there is a lack of behavioral data in the present study, as FSCV experiments were conducted in rats during their light cycle and they tended to sleep throughout the recording; thus, we cannot necessarily conclude that Tyr depletion will have the same effect on dopamine transmission in the NAc during a behavioral task. Indeed, reports from humans drinking an AA beverage suggest that nausea can occur (e.g., Leyton et al., 2000a) and it is unknown whether similar effects might occur in rats. Secondly, the enhancement in Tyr tissue content after BAL compared to saline, which reached significance in the PFC, suggests that the AA load of this mixture was high enough to increase tyrosine levels in the brain. A recent report tested a Phe/Tyr[-] mixture of fewer AAs than used herein that was only slightly less effective than the full complement (Le Masurier et al., 2013). While that study did not employ a control AA mixture, it suggests that using somewhat lower AA loads in future studies may be prudent.

Thus, the present data demonstrate that Tyr depletion reduces spontaneous, phasic dopamine transmission in the NAc, a brain region in which dopamine transients are strongly associated with reward learning and reward-associated cues. This study illuminates a mechanism by which Phe/Tyr[-] impacts dopamine transmission - that is, by reducing the probability of phasic dopamine release events - which may contribute, at least in part, to behavioral changes induced by Phe/Tyr[-] in people.

\section{Acknowledgments}

Authors thank Dr. Paul Kropp for consultation on protocols for the amino acid mixtures, Dr. Chris Wiesen (UNC Odum Institute for Research in Social Science) for statistical consultation and Drs. Margaret Broadwater and Aric Madayag for technical assistance and critical comments on the manuscript.

Funding: This research was funded by the NIH (P60 AA011605, Project \#3), the UNC Bowles Center for Alcohol Studies, R37 AA011852 and R01 AA014874. SCT was supported on a Summer Undergraduate Research Fellowship from UNC Chapel Hill. SJS was supported on 5P60AA011605-17S1 and JMD was supported on T32 AA007471.

\section{References}

Alabi AA, Tsien RW. Synaptic vesicle pools and dynamics. Cold Spring Harb Perspect Biol. 2012; 4:a013680. [PubMed: 22745285]

Barrett SP, Pihl RO, Benkelfat C, Brunelle C, Young SN, Leyton M. The role of dopamine in alcohol self-administration in humans: individual differences. Eur Neuropsychopharmacol. 2008; 18:439_ 447. [PubMed: 18367384]

Bongiovanni R, Newbould E, Jaskiw GE. Tyrosine depletion lowers dopamine synthesis and desipramine-induced prefrontal cortex catecholamine levels. Brain Res. 2008; 1190:39-48. [PubMed: 18082673] 
Borland LM, Shi G, Yang H, Michael AC. Voltammetric study of extracellular dopamine near microdialysis probes acutely implanted in the striatum of the anesthetized rat. J Neurosci Methods. 2005; 146:149-158. [PubMed: 15975664]

Brodnik Z, Double M, Jaskiw GE. Presynaptic regulation of extracellular dopamine levels in the medial prefrontal cortex and striatum during tyrosine depletion. Psychopharmacology. 2013; 227:363-371. [PubMed: 23371490]

Cawley EI, Park S, aan het Rot M, Sancton K, Benkelfat C, Young SN, Boivin DB, Leyton M. Dopamine and light: dissecting effects on mood and motivational states in women with subsyndromal seasonal affective disorder. J Psychiatry Neurosci. 2013; 38:388-397. [PubMed: 23735584]

Day JJ, Roitman MF, Wightman RM, Carelli RM. Associative learning mediates dynamic shifts in dopamine signaling in the nucleus accumbens. Nat Neurosci. 2007; 10:1020-1028. [PubMed: 17603481]

Dreyer JK, Herrik KF, Berg RW, Hounsgaard JD. Influence of phasic and tonic dopamine release on receptor activation. J Neurosci. 2010; 30:14273-14283. [PubMed: 20962248]

Fernstrom MH, Fernstrom JD. Acute tyrosine depletion reduces tyrosine hydroxylation rate in rat central nervous system. Life sciences. 1995; 57:PL97-102. [PubMed: 7630309]

Garris PA, Walker QD, Wightman RM. Dopamine release and uptake rates both decrease in the partially denervated striatum in proportion to the loss of dopamine terminals. Brain research. 1997; 753:225-234. [PubMed: 9125407]

Harmer CJ, McTavish SF, Clark L, Goodwin GM, Cowen PJ. Tyrosine depletion attenuates dopamine function in healthy volunteers. Psychopharmacology. 2001; 154:105-111. [PubMed: 11291999]

Hermans A, Keithley RB, Kita JM, Sombers LA, Wightman RM. Dopamine detection with fast-scan cyclic voltammetry used with analog background subtraction. Anal Chem. 2008; 80:4040-4048. [PubMed: 18433146]

Howard CD, Daberkow DP, Ramsson ES, Keefe KA, Garris PA. Methamphetamine-induced neurotoxicity disrupts naturally occurring phasic dopamine signaling. Eur J Neurosci. 2013; 38:2078-2088. [PubMed: 23574406]

Howard CD, Keefe KA, Garris PA, Daberkow DP. Methamphetamine neurotoxicity decreases phasic, but not tonic, dopaminergic signaling in the rat striatum. J Neurochem. 2011; 118:668-676. [PubMed: 21668447]

Jaskiw GE, Bongiovanni R. Brain tyrosine depletion attenuates haloperidol-induced striatal dopamine release in vivo and augments haloperidol-induced catalepsy in the rat. Psychopharmacology. 2004; 172:100-107. [PubMed: 14586541]

Jaskiw GE, Kirkbride B, Bongiovanni R. In rats chronically treated with clozapine, tyrosine depletion attenuates the clozapine-induced in vivo increase in prefrontal cortex dopamine and norepinephrine levels. Psychopharmacology. 2006; 185:416-422. [PubMed: 16541248]

Jaskiw GE, Kirkbride B, Newbould E, Young D, Durkalski V, Bongiovanni R. Clozapine-induced dopamine release in the medial prefrontal cortex is augmented by a moderate concentration of locally administered tyrosine but attenuated by high tyrosine concentrations or by tyrosine depletion. Psychopharmacology. 2005; 179:713-724. [PubMed: 15682305]

Kelm MK, Boettiger CA. Effects of acute dopamine precusor depletion on immediate reward selection bias and working memory depend on catechol-O-methyltransferase genotype. Journal of cognitive neuroscience. 2013; 25:2061-2071. [PubMed: 23937688]

Kelm MK, Boettiger CA. Age moderates the effect of acute dopamine depletion on passive avoidance learning. Pharmacology, biochemistry, and behavior. 2015; 131:57-63.

Kishida KT, Sandberg SG, Lohrenz T, Comair YG, Saez I, Phillips PE, Montague PR. Sub-second dopamine detection in human striatum. PLoS One. 2011; 6:e23291. [PubMed: 21829726]

Le Masurier M, Oldenzeil W, Lehman C, Cowen P, Sharp T. Effect of acute tyrosine depletion in using a branched chain amino-acid mixture on dopamine neurotransmission in the rat brain.

Neuropsychopharmacology : official publication of the American College of Neuropsychopharmacology. 2006; 31:310-317. [PubMed: 16034439] 
Le Masurier M, Zetterstrom T, Cowen P, Sharp T. Tyrosine-free amino acid mixtures reduce physiologically-evoked release of dopamine in a selective and activity-dependent manner. J Psychopharmacol. 2013; 28:561-569. [PubMed: 24108408]

Leyton M, Dagher A, Boileau I, Casey K, Baker GB, Diksic M, Gunn R, Young SN, Benkelfat C. Decreasing amphetamine-induced dopamine release by acute phenylalanine/tyrosine depletion: A PET/[11C]raclopride study in healthy men. Neuropsychopharmacology. 2004; 29:427-432.

Leyton M, Young SN, Blier P, Baker GB, Pihl RO, Benkelfat C. Acute tyrosine depletion and alcohol ingestion in healthy women. Alcohol Clin Exp Res. 2000a; 24:459-464.

Leyton M, Young SN, Pihl RO, Etezadi S, Lauze C, Blier P, Baker GB, Benkelfat C. Effects on mood of acute phenylalanine/tyrosine depletion in healthy women. Neuropsychopharmacology. 2000b; 22:52-63.

McMillen BA, German DC, Shore PA. Functional and pharmacological significance of brain dopamine and norepinephrine storage pools. Biochem Pharmacol. 1980; 29:3045-3050. [PubMed: 6109535]

McTavish SF, Callado L, Cowen PJ, Sharp T. Comparison of the effects of alpha-methyl-p-tyrosine and a tyrosine-free amino acid load on extracellular noradrenaline in the rat hippocampus in vivo. $\mathrm{J}$ Psychopharmacol. 1999a; 13:379-384. [PubMed: 10667614]

McTavish SF, Cowen PJ, Sharp T. Effect of a tyrosine-free amino acid mixture on regional brain catecholamine synthesis and release. Psychopharmacology. 1999b; 141:182-188. [PubMed: 9952043]

McTavish SF, McPherson MH, Harmer CJ, Clark L, Sharp T, Goodwin GM, Cowen PJ. Antidopaminergic effects of dietary tyrosine depletion in healthy subjects and patients with manic illness. Br J Psychiatry. 2001; 179:356-360. [PubMed: 11581118]

Michael D, Travis ER, Wightman RM. Color images for fast-scan CV measurements in biological systems. AnalChem. 1998; 70:586A-592A.

Moja EA, Lucini V, Benedetti F, Lucca A. Decrease in plasma phenylalanine and tyrosine after phenylalanine-tyrosine free amino acid solutions in man. Life sciences. 1996; 58:2389-2395. [PubMed: 8691983]

Montgomery AJ, McTavish SF, Cowen PJ, Grasby PM. Reduction of brain dopamine concentration with dietary tyrosine plus phenylalanine depletion: an [11C]raclopride PET study. Am J Psychiatry. 2003; 160:1887-1889. [PubMed: 14514507]

Park J, Takmakov P, Wightman RM. In vivo comparison of norepinephrine and dopamine release in rat brain by simultaneous measurements with fast-scan cyclic voltammetry. J Neurochem. 2011; 119:932-944. [PubMed: 21933188]

Robinson DL, Heien ML, Wightman RM. Frequency of dopamine concentration transients increases in dorsal and ventral striatum of male rats during introduction of conspecifics. The Journal of neuroscience : the official journal of the Society for Neuroscience. 2002; 22:10477-10486. [PubMed: 12451147]

Robinson DL, Hermans A, Seipel AT, Wightman RM. Monitoring rapid chemical communication in the brain. Chem Rev. 2008; 108:2554-2584. [PubMed: 18576692]

Robinson DL, Howard EC, McConnell S, Gonzales RA, Wightman RM. Disparity between tonic and phasic ethanol-induced dopamine increases in the nucleus accumbens of rats. Alcoholism, clinical and experimental research. 2009; 33:1187-1196.

Robinson DL, Venton BJ, Heien ML, Wightman RM. Detecting subsecond dopamine release with fastscan cyclic voltammetry in vivo. Clin Chem. 2003; 49:1763-1773. [PubMed: 14500617]

Robinson DL, Wightman RM. Nomifensine amplifies subsecond dopamine signals in the ventral striatum of freely-moving rats. J Neurochem. 2004; 90:894-903. [PubMed: 15287895]

Robinson DL, Zitzman DL, Smith KJ, Spear LP. Fast dopamine release events in the nucleus accumbens of early adolescent rats. Neuroscience. 2011; 176:296-307. [PubMed: 21182904]

Sanghera MK, German DC, Kiser RS, Shore PA. Differences in norepinephrine and dopamine neurotransmitter storage systems. Brain Res Bull. 1979; 4:217-221. [PubMed: 223744]

Schultz W. Dopamine reward prediction-error signalling: a two-component response. Nat Rev Neurosci. 2016 in press. 
Sheehan BD, Tharyan P, McTavish SF, Campling GM, Cowen PJ. Use of a dietary manipulation to deplete plasma tyrosine and phenylalanine in healthy subjects. J Psychopharmacol. 1996; 10:231234. [PubMed: 22302950]

Shnitko TA, Robinson DL. Anatomical and pharmacological characterization of catecholamine transients in the medial prefrontal cortex evoked by ventral tegmental area stimulation. Synapse (New York, NY). 2014; 68:131-143.

Shnitko TA, Robinson DL. Regional variation in phasic dopamine release during alcohol and sucrose self-administration in rats. ACS Chem Neurosci. 2015; 6:147-154. [PubMed: 25493956]

Smith S, Sharp T. Measurement of GABA in rat brain microdialysates using o-phthaldialdehydesulphite derivatization and high-performance liquid chromatography with electrochemical detection. J Chromatogr. 1994; 652:228-233. [PubMed: 8006108]

Sombers LA, Beyene M, Carelli RM, Wightman RM. Synaptic overflow of dopamine in the nucleus accumbens arises from neuronal activity in the ventral tegmental area. The Journal of neuroscience : the official journal of the Society for Neuroscience. 2009; 29:1735-1742. [PubMed: 19211880]

Venugopalan VV, Casey KF, O'Hara C, O'Loughlin J, Benkelfat C, Fellows LK, Leyton M. Acute phenylalanine/tyrosine depletion reduces motivation to smoke cigarettes across stages of addiction. Neuropsychopharmacology. 2011; 36:2469-2476. [PubMed: 21775977]

Volkow ND, Chang L, Wang GJ, Fowler JS, Leonido-Yee M, Franceschi D, Sedler MJ, Gatley SJ, Hitzemann R, Ding YS, Logan J, Wong C, Miller EN. Association of dopamine transporter reduction with psychomotor impairment in methamphetamine abusers. Am J Psychiatry. 2001; 158:377-382. [PubMed: 11229977]

Willuhn I, Wanat MJ, Clark JJ, Phillips PE. Dopamine signaling in the nucleus accumbens of animals self-administering drugs of abuse. Curr Top Behav Neurosci. 2010; 3:29-71. [PubMed: 21161749] 


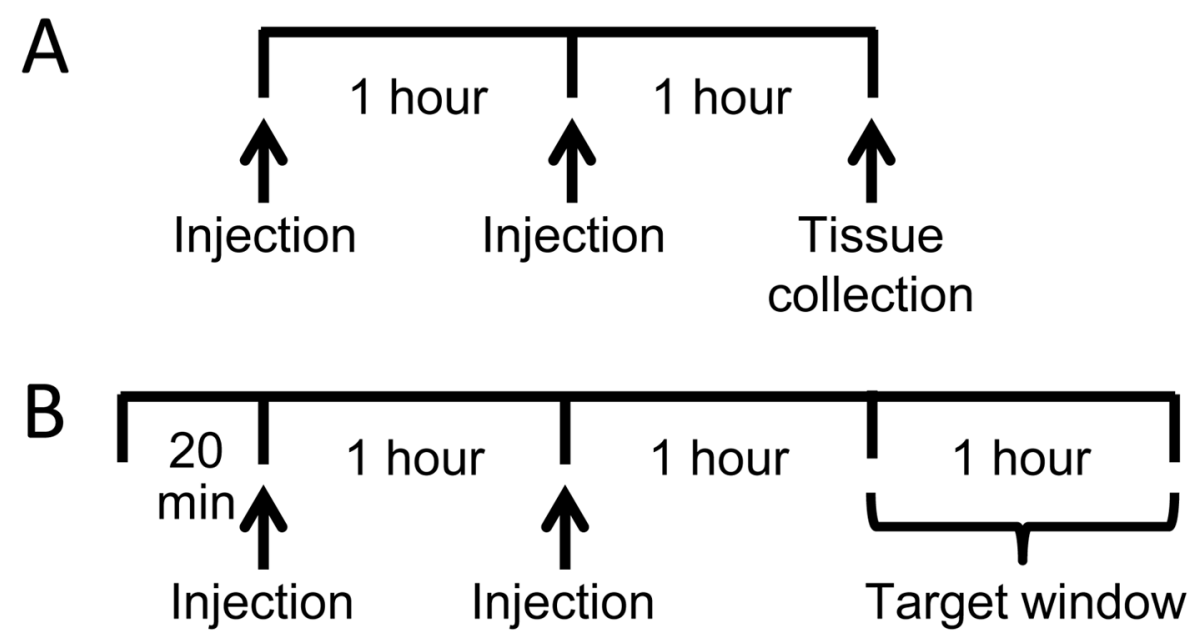

Figure 1.

Experimental timeline for Experiment 1 (panel A) and Experiment 2 (panel B). Baseline levels of dopamine transients were assessed for 20 min before amino acid administration in Experiment 2. The Phe/Tyr[-] or control solutions were administered in two injections, one hour apart, based on McTavish et al. (1999b). One hour after the second injection, tissue was collected for Experiment 1 and the target window to assess changes in dopamine transients from baseline began. 


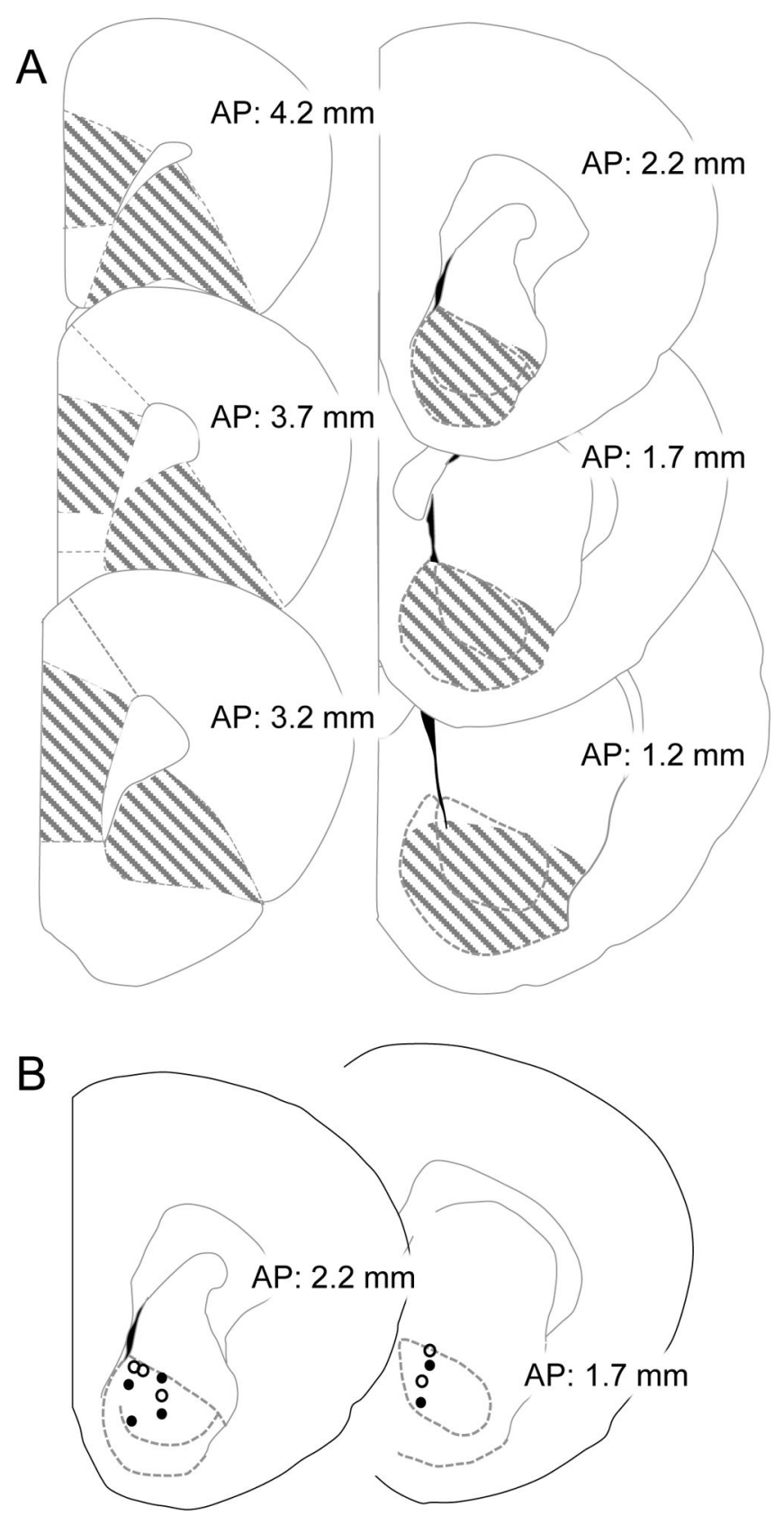

Figure 2.

A: Representative schematic depicting the location of tissue punches taken from the medial prefrontal cortex and orbitofrontal cortex (left) and ventral striatum (right) in Experiment 1. B: Location of carbon fiber electrode placements in the NAc for Experiment 2 estimated from the histological analysis, with open circles representing recordings from the BAL group and filled circles those from the Phe/Tyr[-] group. 
Nucleus accumbens

Prefrontal cortex

A
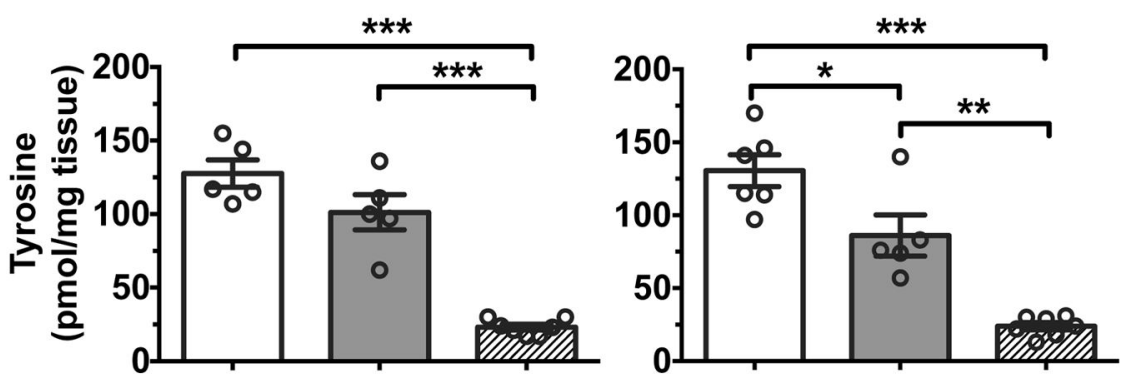

B
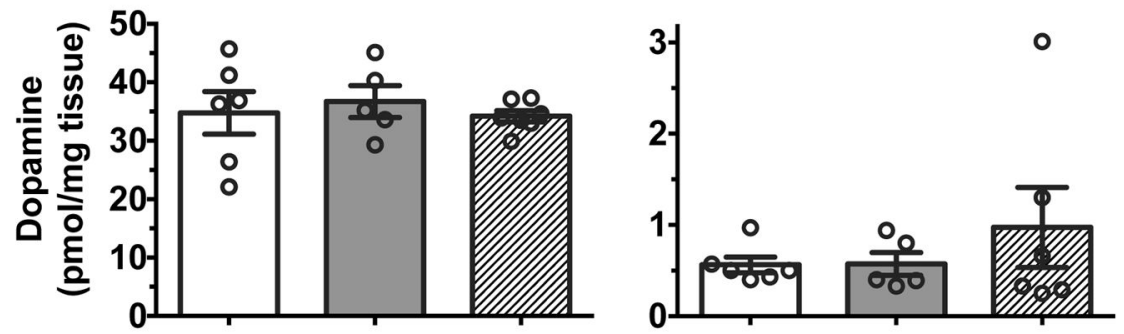

C
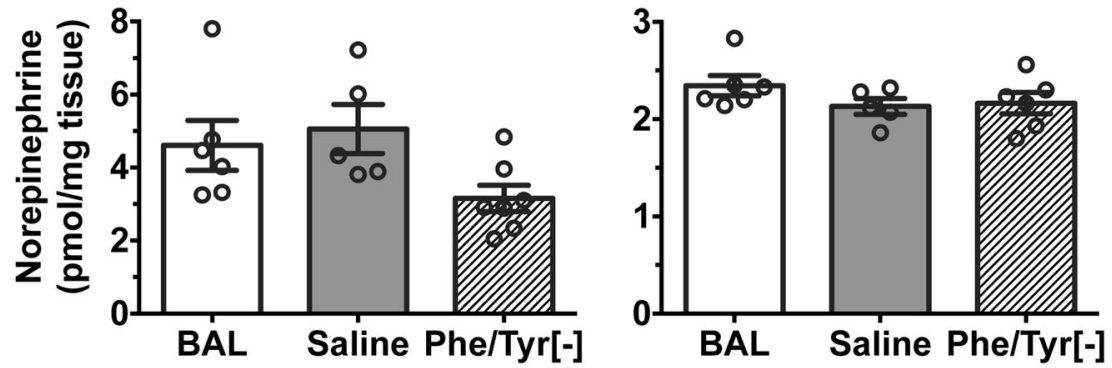

Figure 3.

Phenylalanine and tyrosine depletion mixture reduced tyrosine but not catecholamine content in NAc and PFC. (A) Tyrosine tissue content was reduced after Phe/Tyr[-]. Rat numbers are as follows: NAc BAL $=5$, NAc saline $=5$, NAc Phe/Tyr $[-]=7, \mathrm{PFC} \mathrm{BAL}=6$, PFC saline $=5$, PFC Phe/Tyr[-] $=7 . * \mathrm{p}<0.05, * * \mathrm{p}<0.01, * * * \mathrm{p}<0.001$. Neither dopamine (B) nor norepinephrine (C) tissue content was significantly altered by Phe/Tyr[-]. Rat numbers are as follows: NAc BAL $=6$, NAc saline $=5$, NAc Phe/Tyr[-] $=7, \mathrm{PFC} \mathrm{BAL}=6$, PFC saline $=5$, PFC Phe/Tyr[-] $=6$. 

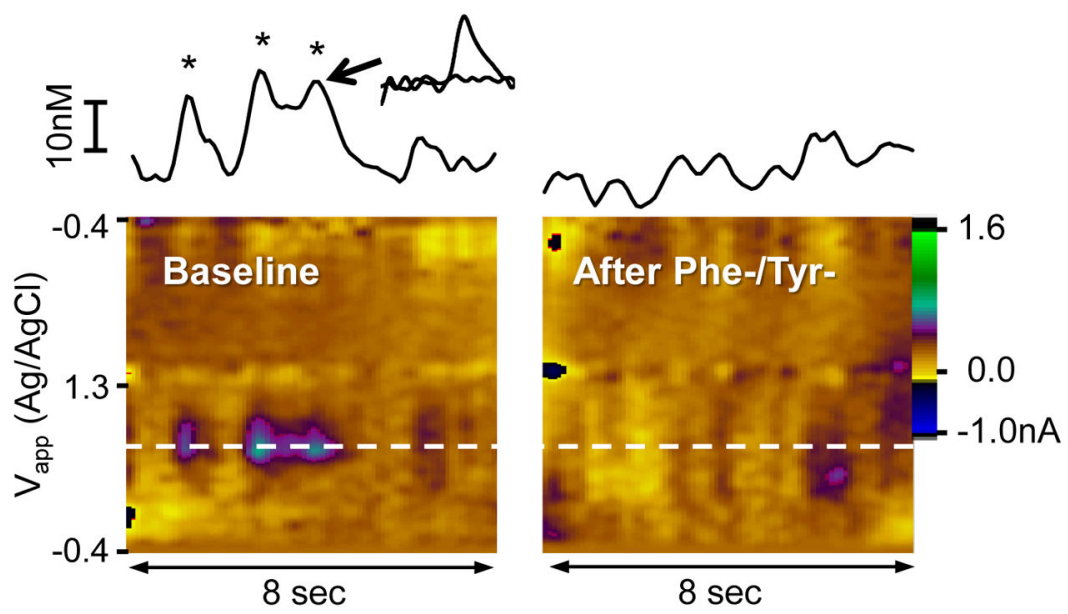

Figure 4.

Examples of dopamine transients in a Phe/Tyr[-]-treated rat as measured with FSCV taken from the in the first 8 seconds of the baseline period (left) and the first 8 second of the target window (right). The color plot shows changes in current (color) at different applied potentials (y-axis) over time (x-axis). (For more explanation of color plots, see Michael et al., 1998). Current at the oxidation potential of dopamine, indicated by the white dashed line, was extracted and converted to concentration via in vitro calibration of the electrode, and the resulting trace is above the color plot. Changes in current confirmed to be due to dopamine oxidation (per the cyclic voltammogram) are indicated with asterisks. The inset shows one of the dopaminergic cyclic voltammograms. 

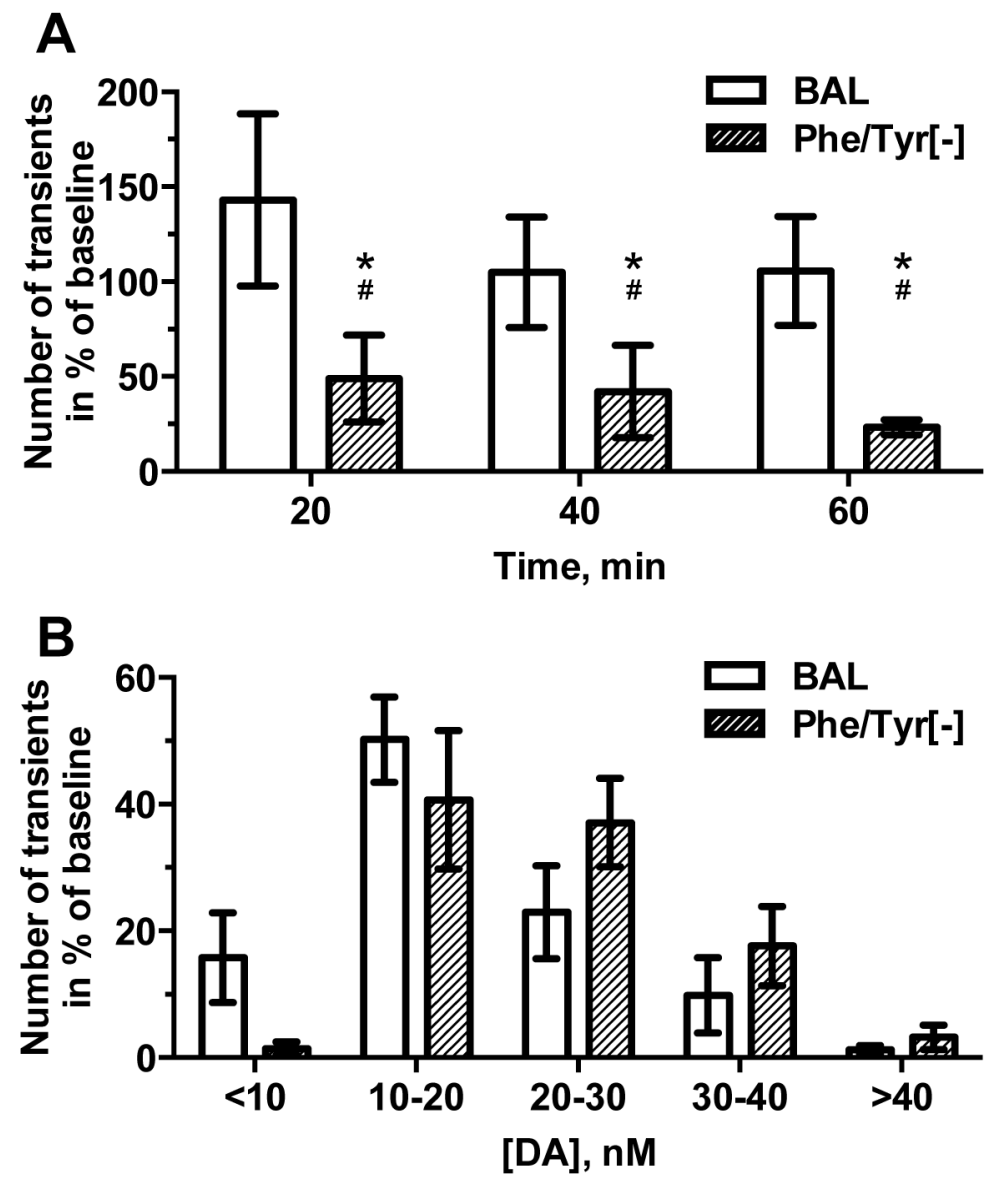

Figure 5.

Administration of Phe/Tyr[-] reduced the number but not amplitude of dopamine release events in the NAc. (A) The frequency of dopamine transients during the three 20 -min bins of the target window relative to the 20-min baseline (pre-injection) period. Phe/Tyr[-] reduced the number of transients detected by more than $50 \%$, while no significant change was observed in the BAL group. * Different from baseline, $\mathrm{p}<0.001$; \# different from BAL, $\mathrm{p}<$ 0.05. (B) The proportion of transients during the target window at various concentrations was compared between groups, and Phe/Tyr[-] did not significantly alter amplitude $\left([\mathrm{DA}]_{\max }\right)$ of transients. Data are $n=6 /$ group. 